\title{
Parity-broken ground state for the spin-1 pyrochlore antiferromagnet
}

\author{
Yasufumi Yamashita ${ }^{1}$, Kazuo Ueda ${ }^{1,2}$ and Manfred Sigrist ${ }^{3,4}$ \\ ${ }^{1}$ Institute for Solid State Physics, University of Tokyo, 5-1-5 Kashiwa-no-ha, Kashiwa, Chiba 277-8581, Japan \\ ${ }^{2}$ Advanced Science Research Center, Japan Atomic Energy Research Institute, Tokai, Ibaraki 319-1195, Japan \\ 3 Yukawa Institute for Theoretical Physics, Kyoto University, Kyoto 606-8502, Japan \\ ${ }^{4}$ Theoretische Physik, ETH-Hönggerberg CH-8093 Zürich, Switzerland
}

(Received November 20, 2018)

\begin{abstract}
The ground-state properties of the spin-1 pyrochlore antiferromagnet are studied by applying the VBS-like tetrahedron-unit decomposition to the original spin system. The symmetrization required on every vertex is taken into account by introducing a ferromagnetic coupling. The pairwise effective Hamiltonian between the adjacent tetrahedrons is obtained by considering the next nearest neighbor and the third neighbor exchange interactions. We find that the transverse component of the spin chirality exhibits a long-range order, breaking the parity symmetry of the tetrahedral group, while the chirality itself is not broken.
\end{abstract}

PACS numbers: 75.10.Jm, 75.40.Cx, 75.10.-b

The pyrochlore lattice, the network of the corner sharing tetrahedrons or a fcc-array of tetrahedrons, is a typical example of a three-dimensional(3D) frustrated system, which is found in a number of materials, such as spinels, pyrochlores and C15-type Laves phase. To study effects of the geometric frustrations and the resultant enhanced spin fluctuations, the pyrochlore spin systems have been investigated intensively. For the spin- $1 / 2$ antiferromagnetic(AF) Heisenberg model, the quantum spinliquid ground state is proposed based on the series expansion at finite temperature [1], but concerning the properties of the low-lying spin-singlet states below the spin gap, a consistent picture has not emerged yet $[2] 5]$.

For the spin-1 case, numerical methods powerful for the spin- $1 / 2$ systems become difficult to obtain definitive

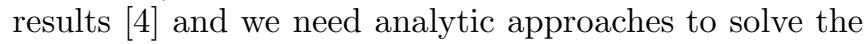
problem. We proposed the idea of the tetrahedron-unit decomposition of the pyrochlore lattice, which is a natural generalization of the valence-bond-solid(VBS) state approach developed for the 1D spin-1 systems [7]. In this approach the ground states of the fundamental unit, corresponding to the valence bond for the VBS case, is the tetrahedron spin singlets which form the two-dimensional $E$ representation of the tetrahedral group $\left(T_{d}\right)$. In consequence, the constructed VBS-type wavefunctions define the variational spin-singlets manifold with a macroscopic degeneracy, to which the ground state of the original problem may be continued adiabatically. In this situation, it is essential to investigate how the ground state in the thermodynamic limit is stabilized from a collection of spin singlets by lifting the degeneracy.

In the previous paper [7], we examined the magnetoelastic couplings between the tetrahedron-singlets and the local lattice distortion of the $E$ modes on the same tetrahedron as the source of lifting the degeneracy. As a result we have found that a Jahn-Teller mechanism driven by the non-magnetic spin degrees of freedom gives rise to the structural phase transition. The local lattice distortion is given by $Q_{v}$ mode of $T_{d}$. Actually the uni- form $Q_{v}$ distortion compressed along the $c$-axis is consistent with the cubic to tetragonal structural phase transition without accompanying any magnetic order, observed in the spin- 1 spinel-type antiferromagnets, $\mathrm{ZnV}_{2} \mathrm{O}_{4}$ and $\mathrm{MgV}_{2} \mathrm{O}_{4}$. This scenario can explain, at least qualitatively, the experimental results around the structural transition temperature.

As another way of lifting the degeneracy, in this letter, we study the case where the inter-tetrahedron interactions, the next nearest neighbor and the third neighbor spin-spin interactions, are relevant. In particular, we will discuss the properties of the order parameters of the obtained ground state. Since the tetrahedron-singlets may be labeled by the spin chirality, a chiral ordered state is an interesting possibility. However, as long as physically reasonable interactions, such as spin exchange interaction or the dipole interaction, are considered, we find that it is not the chirality but its transverse component that shows the long-range order.

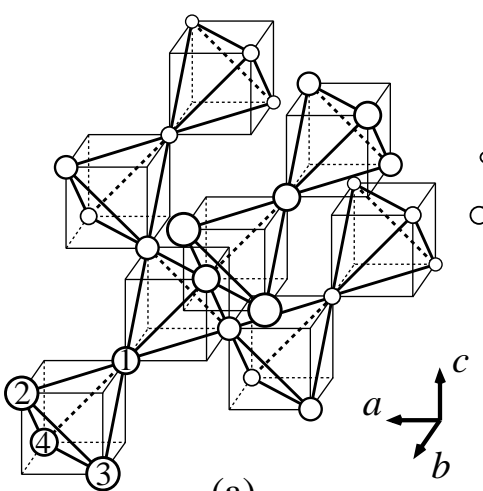

(a)

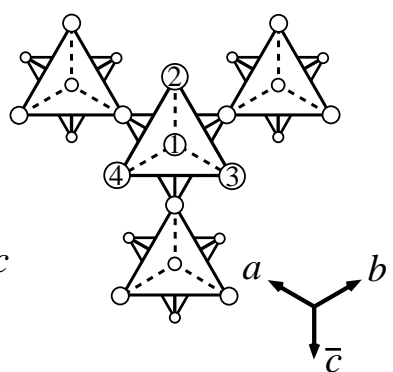

(b)
FIG. 1. The primitive cell of the pyrochlore lattice (a) and its projected view from the $[11 \overline{1}]$-axis (b). The small cubes in figure (a) are the guide for eyes with one-sixteenth volume of the unit cell.

Following the 1D VBS approach, we break up the orig- 
inal spin- 1 objects (denoted by $\vec{S}_{i}$ ) into the two spin- $1 / 2$ ones $\left(\vec{s}_{i a}\right.$ and $\left.\vec{s}_{i b}\right)$ and introduce a ferromagnetic Hund couplings between them $\left(-J_{F}\right)$, which serve as the symmetrization. To project out the singlet sector of the composite spins completely it is necessary to take the $J_{F} \rightarrow \infty$ limit, however in nature a strong but finite $J_{F}$ is sufficient. The pyrochlore lattice may be decomposed into the $A$ and $B$ types of bonds, which form the upward and downward tetrahedrons as shown in Fig. 1 (b). Because of the symmetrization it is allowed to consider that the $1 / 2$-spins with $a(b)$ index construct the network of the $A(B)$ bonds without loss of generality. In this decomposite-spin representation, the original AF Heisenberg model can be rewritten as follows in the strong ferromagnetic coupling limit $\left(J_{F} \rightarrow \infty\right)$;

$$
\begin{aligned}
\mathcal{H}_{\text {dec. }} & =4 J \sum_{<i, j>\in A} \vec{s}_{i a} \cdot \vec{s}_{j a}+4 J \sum_{<i, j>\in B} \vec{s}_{i b} \cdot \vec{s}_{j b} \\
& -J_{F} \sum_{i} \vec{s}_{i a} \cdot \vec{s}_{i b} .
\end{aligned}
$$

In the $J_{F}=0$ limit, the ground states of Eq. (1) are given by;

$$
\left|\Psi_{0}\right\rangle=\prod_{k=1}^{N / 2}\left(\alpha_{k}|u\rangle_{k}+\beta_{k}|v\rangle_{k}\right),
$$

with arbitrary complex parameters $\alpha_{k}$ and $\beta_{k}$ keeping $\left|\alpha_{k}\right|^{2}+\left|\beta_{k}\right|^{2}=1$, where $N$ is the number of sites and $k$ specifies a tetrahedron. Here the orthonormal basis, $\{|u\rangle,|v\rangle\}$ defined for a single tetrahedron, are the total spin-singlet states with $\vec{s}_{1 x}+\vec{s}_{2 x}=\vec{s}_{3 x}+\vec{s}_{4 x}=0$ or 1, respectively, $(x=a, b)$ (see Fig. 2). These tetrahedron singlets are the non-magnetic doublets belonging to the $E$ representation of $T_{d}$ and the $2^{N / 2}$-fold degenerate manifold defined by Eq.(2) is expected to be adiabatically continued to the low-energy sector of the original model. When representing the symmetry operation of $T_{d}$ in this singlet subspace, $\{|u\rangle,|v\rangle\}$ real-basis diagonalizes the parity operations with respect to the bonds vertical to the $c$-axis. On the other hand, the chirality basis [7, which is defined by $|R\rangle=(|u\rangle-i|v\rangle) / \sqrt{2}$ and $|L\rangle=|R\rangle^{*}$, diagonalizes the four distinct $C_{3}$ operations with the eigenvalues of $\omega=(-1+\sqrt{3} i) / 2$ and its complex conjugate $\omega^{*}$, respectively.

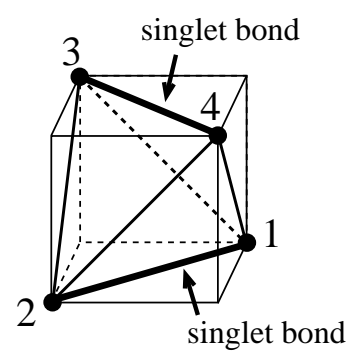

$|u\rangle$-state

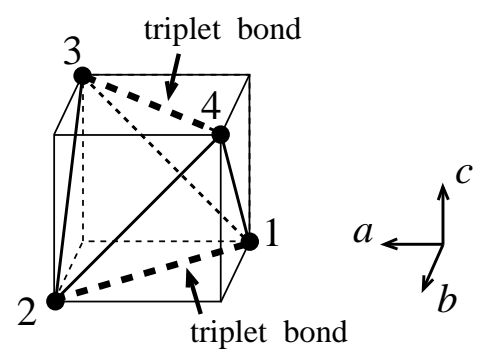

$|v\rangle$-state
FIG. 2. The schematic representations of the tetrahedron-singlet states, $|u\rangle$ and $|v\rangle$.
We proceed to the next step of lifting the $2^{N / 2}$-fold degeneracy of Eq. (2). When considering the pure twotetrahedron problem, we can easily see that the ferromagnetic coupling $J_{F}$ alone does not lift the degeneracy. Therefore the pairwise perturbation between the two adjacent tetrahedrons caused by $J_{F}$ never fixes the original $2 \times 2$-fold degeneracy. ¿From the viewpoint of geometry, this means that the relative rotation of two tetrahedrons is not fixed by only $J_{F}$. The degeneracy is partly lifted by considering three tetrahedrons and eventually the degeneracy will be lifted for the entire lattice. However energy scales of the lifting of degeneracy are expected to be small and hierarchical [5]. This problem is interesting but more academic since in nature there are other couplings to lift the degeneracy. In [7] we have considered the local coupling with lattice distortion which leads to the structural phase transition. As another source of lifting the degeneracy we can introduce longer range interactions which are relevant to lift the degeneracy already for a pair of tetrahedrons.

In order to fix the relative rotation of the two adjacent tetrahedrons, we include the next nearest neighbor $\left(J_{1}\right)$ and the third neighbor interactions $\left(J_{2}\right)$. In spinels, $J_{2}$ is expected to be important because of the existence of a superexchange-path through the oxygens on a single plane. The arrangement of these interactions are shown in Fig. 3. By the second-order perturbation in $-J_{F}, J_{1}$, and $J_{2}$ within the degenerate subspace spanned by Eq.(2), we obtain the pairwise effective Hamiltonian between the tetrahedron-singlet states on the adjacent tetrahedrons as follows;

$$
\begin{aligned}
\mathcal{H}_{\text {eff }}= & \frac{1}{2}\left(J_{2}-J_{1}\right)\left(J_{F}-4 J_{2}\right) \\
& \times \sum_{<k_{a}, k_{b}>}\left(\tau_{k_{a}}^{+} \tau_{k_{b}}^{-}+\tau_{k_{a}}^{-} \tau_{k_{b}}^{+}\right)+C_{1},
\end{aligned}
$$

where the coupling is completely the same for the four distinct directions of pairs. In Eq.(3),

$$
\begin{aligned}
C_{1}=-\frac{N}{64}\left\{\left(J_{F}-4 J_{2}\right)^{2}\right. & +2\left(J_{F}-4 J_{1}\right)^{2} \\
& \left.+10\left(4 J_{2}-4 J_{1}\right)^{2}\right\},
\end{aligned}
$$

and the Pauli matrix $\vec{\tau}_{k}$, describing the two-dimensional spin-singlet space on the $k$ th tetrahedron, is defined by using the chirality basis as,

$$
\begin{aligned}
\tau^{z} & =|R\rangle\langle R|-| L\rangle\langle L|, \\
\tau^{+} & =|R\rangle\left\langle L\left|, \quad \tau^{-}=\right| L\right\rangle\langle R| .
\end{aligned}
$$

In fact, we can show that the universality class of the effective Hamiltonian is $X X Z$ type by using a symmetry consideration as follows. First, the $\pm 2 \pi / 3$ rotation, $C_{3}$ 
and $C_{3}^{-1}$, around the axis joining the centers of two tetrahedrons leads to the conservation of their total chirality. Second, the $|R\rangle$ and $|L\rangle$ states are mutually conjugate by the time-reversal symmetry. Therefore we can conclude that the derived effective interaction has the $X X Z$-type symmetry of the pseudo $\vec{\tau}$-spins and that the Ising term must vanish in the even-order perturbations. It should be noted that the second-order perturbation gives the leading terms, since we start from the non-magnetic zeroth order states.

In order to estimate the higher-order perturbation terms concerning $J_{F}$ within the pairwise treatment, we consider the infinite $J_{F}$ limit by considering the original spin-1 object on the sharing top vertex of the two tetrahedrons. In this case, the effective Hamiltonian obtained by the first-order perturbation is given by,

$$
h_{k_{a}, k_{b}}=\frac{1}{2.25}\left(J_{2}-J_{1}\right)\left(\tau_{k_{a}}^{+} \tau_{k_{b}}^{-}+\tau_{k_{a}}^{-} \tau_{k_{b}}^{+}\right)+C_{2},
$$

with $C_{2}=\left(J_{2}+2 J_{1}\right) / 9$, which is just $8 / 9$ of the coefficient of the first order term of Eq. (3) in $J_{F}$. The $\vec{\tau}$ matrixes here are defined concerning the bottom triangles, which are related to the $\vec{\tau}$ matrix in Eq. (3) through the relations like $|R\rangle=\left(\left|\uparrow_{R}\right\rangle\left|\downarrow_{V}\right\rangle-\left|\downarrow_{R}\right\rangle\left|\uparrow_{V}\right\rangle\right) / \sqrt{2}$, where $\left|\uparrow_{R}\right\rangle$ is the doublet states with $R$ chirality about the bottom triangle and $\left|\uparrow_{V}\right\rangle$ represents the spin state on the top vertex. It should be noted that such decompositions cannot be extended consistently all over the lattice. Here we have shown it to illustrate the generic form of the pairwise effective interaction in the strong $J_{F}$ limit.

Comparison of Eq. (3) and Eq. (5) suggests that higher order effects of $J_{F}$ only renormalize the strength of the pairwise interaction. Therefore, we assume that the effective Hamiltonian to describe the low energy part of the original Heisenberg model with next nearest neighbor $\left(J_{1}\right)$ and third neighbor interactions $\left(J_{2}\right)$ is given as follows, by using the spin-1/2 pseudospin operator, $\vec{\tau}_{k}$, defined for the $k$ th tetrahedron;

$$
\mathcal{H}_{e f f}=c J^{\prime} \sum_{\left\langle k_{a}, k_{b}\right\rangle}\left(\tau_{k_{a}}^{+} \tau_{k_{b}}^{-}+\tau_{k_{a}}^{-} \tau_{k_{b}}^{+}\right),
$$

where $J^{\prime}=J_{2}-J_{1}$ with a positive $c$. Since both of the upward (labeled by $k_{a}$ ) and downward $\left(k_{b}\right)$ tetrahedrons form the distinct fcc lattice structure, Eq. (6) is the spin-1/2 XY model on ZnS type bipartite lattice with coordination number $z=4$.

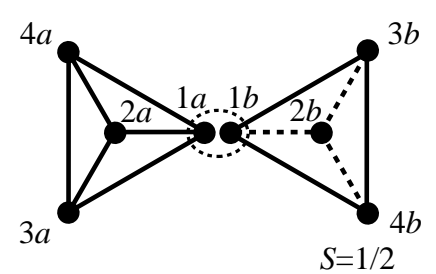

$$
\begin{aligned}
J & :-, \cdots \\
-J_{F}: \cdots & \cdots \\
J_{1}: & (2 a, 3 b),(2 a, 4 b),(3 a, 2 b), \\
& (3 a, 4 b),(4 a, 2 b),(4 a, 3 b) \\
J 2 & :(2 a, 2 b),(3 a, 3 b),(4 a, 4 b)
\end{aligned}
$$

FIG. 3. The decomposite-spin representation of the Heisenberg model $\left(J\right.$ and infinite $\left.J_{F}\right)$ and the next-nearest $\left(J_{1}\right)$ and third-neighboring interactions $\left(J_{2}\right)$ are shown for the pairwise $\left(k_{a}, k_{b}\right)$ tetrahedrons.
Since the sign of the coupling constant of the XY model on a bipartite lattice can be converted by the $\pi$-rotation around the $z$-axis concerning one of the two sublattices, in the following we discuss the case with the negative interaction. For the spin- $1 / 2$ case with $N$ sites, it is exactly shown that the ground state is unique with $S_{\text {tot }}^{z}=0$ by using the Perron-Frobenius theorem [10]. As one can imagine naively, the state with $S_{t o t}=N / 2$ and $S_{\text {tot }}^{z}=0$ is a good zeroth order approximation. In fact, the Gutzwiller-type wavefunction constructed from this state is shown to be an extremely good variational state [11,12. When applying their results for our $z=4$ case, the square of the long-range order (LRO) and the shortrange magnetic correlation are given by;

$$
\begin{aligned}
& \left\langle M^{x 2}\right\rangle / N^{2}=2^{15} \cdot 3^{4} \cdot 7^{-8}=0.4604 \cdots \\
& \left\langle\tau_{k}^{z} \cdot \tau_{k+\delta}^{z}\right\rangle=-1 / 7
\end{aligned}
$$

where $\vec{M}=\sum_{k=1}^{N} \vec{\tau}_{k}$ and $\vec{\tau}_{k+\delta}$ means the nearest neighbor pseudospin next to the $\vec{\tau}_{k}$ pseudospin. In the XY model, the Hamiltonian has the $\mathrm{U}(1)$ symmetry about the rotation around the $z$ axis in the spin space. Therefore the direction of the order parameter in the $\tau^{x}-\tau^{y}$ plane cannot be determined even for the infinite system. The U(1) symmetric general order parameters, $\vec{M}(\theta)$, are defined by the ground-state expectation value of the following operator;

$$
\hat{M}(\theta)=\left(M^{x} \cos \theta, M^{y} \sin \theta, 0\right),
$$

where $\left\langle M^{x}\right\rangle=\left\langle M^{y}\right\rangle=M>0$. Concerning every local tetrahedron, $\hat{M}(\theta)$ belongs to the $E$ representation of $T_{d}$.

Although the effective Hamiltonian, Eq. (6), has the continuous U(1) symmetry as represented by Eq. (9), the original model possesses only the finite group $\left(T_{d}\right)$ symmetry. The $U(1)$ symmetry is fictitious one valid only in the lowest-order treatments with the pseudo-spin representation. Suppose that we take account of the higher order interactions like biquadratic term and others, then this spurious U(1) symmetry should be broken. In order to understand how this lowering of the symmetry acts, we make use of the Ginzburg-Landau type argument by expanding the free energy as a function of the order parameter $\vec{M}(\theta)$, actually by its magnitude $M$ and phase $\theta$, just around the second-order transition point. The U(1) symmetry, obtained by the perturbation, corresponds to the second-order expansion of the free energy by $\vec{M}(\theta)$. This is because, on every tetrahedron, the product representation $E \times E\left(=A_{1}+A_{2}+E\right)$ contains the unique totally symmetric one $\left(A_{1}\right)$, which is a constant independent of $\theta$. Now we proceed to higher order corrections. Since under the time-reversal symmetry only $\tau^{z}$ changes the sign, while both $\tau^{x}$ and $\tau^{y}$ remain unchanged, the third-order expansion gives the next leading term, introducing the anisotropy to fix $\theta$. By calculating the third 
order invariant, which is obtained by reducing the product representation $E \times E \times E$, we find that the anisotropy is proportional to $\cos 3 \theta$. Thus we get a set of the stable states, $\{\vec{M}(\pi), \vec{M}( \pm \pi / 3)\}$ or $\{\vec{M}(0), \vec{M}( \pm 2 \pi / 3)\}$, depending on the sign of the $M^{3}$ term. These two sets only differ in the overall sign and are essentially the same.

Let us illustrate the symmetry property of the order parameter by taking $\vec{M}(0)=\sum_{k=1}^{N}\left\langle\tau_{k}^{x}\right\rangle=$ $\sum_{k=1}^{N}\left\langle\mid u_{k}\right\rangle\left\langle u_{k}|-| v_{k}\right\rangle\left\langle v_{k} \mid\right\rangle$ as an example. The nonvanishing long-range correlation between $\tau_{k}^{x}$ and $\tau_{k^{\prime}}^{x}$ results in the different population of the $|u\rangle$ and $|v\rangle$ states on a tetrahedron. Therefore the parity symmetry concerning the bonds vertical to the $c$-axis is broken in the ground state, since the $|u\rangle$ and $|v\rangle$ states are characterized by the odd and even parity concerning these bonds, respectively. Three different values of $\theta$ in each set of order parameters correspond to the three equivalent choice of the cubic principal axis; $\vec{M}(2 \pi / 3)$ and $\vec{M}(-2 \pi / 3)$ characterizes the break-down of the parity symmetry concerning the bonds vertical to $b$ and $c$ axis, respectively. These three states form a domain structure in a real system.

Now we extend the present results for positive $J_{F}$ also and summarize the ground-state properties of Eq. (6) by taking $c$-axis as a principal axis $(\theta=0, \pi)$. Depending on the sign of $J^{\prime}=J_{2}-J_{1}$, the uniform $\left(J^{\prime}<0\right)$ or the staggered signed $\left(J^{\prime}>0\right)$ summation of the $\left\langle\tau_{k}^{x}\right\rangle$ all over the tetrahedrons defines the order parameter. In the classical picture, for negative $J^{\prime}$ the ground state shows the ferromagnetic LRO of the transverse components of the spin chirality $|u\rangle(|v\rangle)$ when $\theta=0(\pi)$, while for positive $J^{\prime}$ the antiferromagnetic LRO of the $|u\rangle$ and $|v\rangle$ states. For a local tetrahedron problem the compressed and elongated $Q_{v}$ mode along the $c$-axis stabilize the $|u\rangle$ and $|v\rangle$ states, respectively $[7]$. Therefore, for the ferromagnetic $J^{\prime}$ model the order parameter, $\vec{M}(0)(\vec{M}(\pi))$, induces the uniform $Q_{v}$ lattice distortion with $c / a<1$ $(>1)$. On the other hand, for the $\mathrm{AF} J^{\prime}$ the staggered $Q_{v}$ distortion is induced. Accordingly, under the assumption that the inter-tetrahedron interactions are more important than the local magneto-elastic couplings, the intertetrahedron couplings $\left(J_{1}\right.$ and $\left.J_{2}\right)$ lift the many-fold degeneracy of the spin-singlet manifold by the second-order phase transition. However, introduction of the magnetoelastic coupling, even for a weak case, will induce a small first order structural distortion, leading eventually to a weak first order phase transition.

In conclusion, we have studied the ground-state properties of the spin-1 pyrochlore antiferromagnet by using the tetrahedron-unit decomposition of the pyrochlore lattice. In this approach, we assume that the spinsinglet manifold spanned by the VBS-type wavefunction well-describes the low-energy physics and the essential point is how the degeneracy is lifted. We have inves- tigated the case where this degeneracy is lifted by the inter-tetrahedron interactions caused by the next nearest neighbor and the third neighbor interaction, which produce the the pairwise effective Hamiltonian of the $X Y$ type between the pseudospin operators describing the tetrahedron singlets. To break the spurious U(1) symmetry of the effective model, we have considered the higher order anisotropic term based on the symmetry property $(E)$ of the order parameter. It has turned out that the parity-broken ground state emerges through the secondorder phase transition.

Lastly we briefly comment on the relation between the present results and the numerical results for the pure spin-1 Heisenberg model [4]. The singlet ground states of a single spin-1 tetrahedron problem are three dimensional with $A_{1}+E$ irreducible representations. When we define these spin-singlet states, with $\vec{S}_{1}+\vec{S}_{2}=\vec{S}_{3}+\vec{S}_{4}=0,1$, and 2 , by $|a\rangle,|b\rangle$, and $|c\rangle$, respectively, the orthonormal basis of the $E$ representation are given by $\{|U\rangle,|V\rangle\}=$ $\{|b\rangle,(-2|a\rangle+\sqrt{5}|c\rangle) / 3\}$. Considering the fact that the symmetrization on every vertex does not affect the local symmetry properties, the parity-broken symmetry of the ground state, obtained by using the decomposite-spin representation, may correspond to the $|U\rangle$ and $|V\rangle$ states in the spin-1 picture. Since Koga et. al suggested the possibility of the new spin-gap ground state characterized by the $|U\rangle$ state around the isotropic Heisenberg limit [4], our result seems to be consistent with their result.

We are grateful to H. Tsunetsugu, A. Koga, and N. Kawakami for useful comments and discussions. Y.Y. is supported by the Japan Society for the Promotion of Science.

[1] B. Canals and C. Lacroix, Phys. Rev. B 61, 1149 (2000).

[2] A. Harris and A. Berlinsky, and C. Bruder, J. Appl. Phys. 69, 5200 (1991).

[3] M. Isoda and S. Mori, J. Phys. Soc. Jpn. 67, 4022 (1988).

[4] A. Koga and N. Kawakami, Phys. Rev. B 63, 144432 (2001)

[5] H. Tsunetsugu, J. Phys. Soc. Jpn. 70, 640 (2001).

[6] I. Affleck, T. Kennedy, E. H. Lieb, and H. Tasaki, Phys. Rev. Lett. 59, 799 (1987).

[7] Y. Yamashita and K. Ueda, Phys. Rev. Lett. 85, 4960 (2000).

[8] H. Kawamura, Phys. Rev. Lett. 68, 3785 (1992).

[9] K. Ohgushi, S. Murakami, and N. Nagaosa, Phys. Rev. B 62, R6065 (2000).

[10] D. Mattis, Phys. Rev. Lett. 42, 1503 (1979).

[11] M. Suzuki and S. Miyashita, Can. J. Phys. 56, 902 (1978).

[12] J. Oitmaa and D. Betts, Can. J. Phys. 56, 897 (1978). 